\title{
Review
}

\section{Molecular mechanisms of the physiological functions of the aryl hydrocarbon (dioxin) receptor, a multifunctional regulator that senses and responds to environmental stimuli}

\author{
By Yoshiaki FuJII-KurIYAmA*1,† and Kaname KAWAJIRI*2 \\ (Communicated by Takao SEKIYA, M.J.A.)
}

\begin{abstract}
The aryl hydrocarbon receptor (AhR) was originally identified as a ligandactivated transcription factor that is involved in the induction of xenobiotic-metabolizing Cytochrome P4501A1 (CYP1A1). For several decades, AhR has been studied in relation to toxicology and pharmacology. With recent discoveries on novel AhR functions, AhR research has expanded into multiple aspects of physiology, such as reproduction, innate immunity and tumor suppression. In this review, we summarize and discuss recent progress in mechanistic and functional studies on AhR with particular emphasis on physiological processes.
\end{abstract}

Keywords: bHLH-PAS transcription factor, innate immunity, reproduction, tumor suppression, ubiquitylation, environmental stimuli

\section{Introduction: A brief history of AhR research}

Studies on the aryl hydrocarbon receptor (AhR, also known as dioxin receptor) began about 50 years ago when drug-metabolizing enzymes were found to be induced by polycyclic aromatic hydrocarbons (PAHs), such as 3-methylchoranthrene (3MC) and 2,3,7,8-tetrachlorodibenzo- $p$-dioxin (TCDD). Around 1960 , three research groups independently reported that drug-metabolizing enzymes were induced in rat livers. ${ }^{1-4)}$ Subsequently, in 1962, Omura and Sato identified a microsomal component that gave a unique absorption spectrum with a peak at $450 \mathrm{~nm}$ when bound to $\mathrm{CO}$ in the presence of NADPH, as a hemoprotein and designated this newly discovered hemoprotein Cytochrome P4505) and Cooper et al. revealed that this hemoprotein was a drug-metabolizing enzyme using a photo-reactivation method in which the CO-inhibited drug-metabolizing activity was reversed by treatment with flash light.6)

*1 Institute of Molecular and Cellular Biosciences, University of Tokyo, Tokyo, Japan and Medical Research Institute, Tokyo Medical Dental University, Tokyo, Japan.

*2 Research Institute for Clinical Oncology, Saitama Cancer Center, Saitama, Japan.

$\dagger$ Correspondence should be addressed: Y. Fujii-Kuriyama, 518-7 Honkomagame, Bunkyo-ku, Tokyo 113-0021 Japan (e-mail: y.k_fujii@nifty.com).
In 1976, Poland et al. identified a cytoplasmic factor that bound TCDD with high affinity and called this novel protein AhR. ${ }^{7)}$ Based on both the behavior of AhR in induced cells and the genetics of the inducibility of the drug-metabolizing activity, they proposed that this cytoplasmic factor was involved in inducing the drug-metabolizing P450. ${ }^{8}$ ) In the early 1980s, cDNA clones of phenobarbital-inducible and 3MC-inducible P450s, and subsequently, their genomic clones were isolated by the then-newly-developed molecular cloning technology. 9),10) Biochemical and molecular biological approaches used a reporter gene, in which the chloramphenicol acetyltransferase structural gene was under the control of the rat $P 450 \mathrm{c}$ (CYP1A1) promoter, to identify regulatory sequences within the promoter that induce the reporter gene in response to $3 \mathrm{MC}$ and designated these sequences XRE (Xenobiotic Responsive Element, subsequently named DRE: Drug Responsive Element, or AhRE). ${ }^{11}$ Gel mobility shift assays (GMSA) revealed that an XRE-binding factor translocates from the cytoplasm into the nucleus upon TCDD treatment. In addition, this XRE-binding factor contained TCDD as a ligand, suggesting that the XRE-binding factor was either AhR or contained AhR as a component. ${ }^{12)}$ Subsequently, studies on the mechanisms that regulate mouse Cyp1A1 gene expression revealed a similar 
array of enhancer sequences in the promoter, which were designated DRE. ${ }^{13)}$

Using the XRE-binding property of AhR and a partial N-terminal 20 amino acid sequence determined by Bradfield et al. of the purified mouse AhR fragment, which was photo-labeled with a TCDD derivative, ${ }^{14)}$ we isolated a cDNA clone of $\mathrm{AhR}$ from a cDNA library of the mouse cell line Hepa-1. Using this clone, we were able to determine the amino acid sequence of AhR and show that AhR has a bHLH motif and a PAS ${ }^{15)}$ domain (an amino acid sequence that is conserved among the Per, Arnt and Sim proteins). Our findings were subsequently confirmed by similar data from Bradfield et al. ${ }^{16)}$ The isolation of the AhR cDNA clone furthered our understanding of the molecular mechanisms that regulate the transcription of AhR target genes in response to xenobiotics including TCDD, and the toxicological effects of TCDD that are mediated by AhR. ${ }^{17), 18)}$

In 1995, Gonzalez et al. first described AhRdeficient mice that were generated by homologous recombination, ${ }^{19)}$ and two other research groups including ours subsequently reported the generation of these mice. ${ }^{20), 21)}$ Experiments using these mutant mice clearly demonstrated that AhR mediates many pharmacological and toxicological effects, including the induction of drug-metabolizing CYP1A1, teratogenesis, immune suppression, tumor promotion, and liver damage caused by TCDD, as the AhR-deficient mice were resistant to these effects. ${ }^{21)}$-23) Until the turn of the century, most if not all of the AhR research work had focused on elucidating the molecular mechanisms by which AhR mediated the TCDDinduced pharmacological and toxicological effects, which are detrimental to most living organisms. ${ }^{17), 18 \text { ) }}$

Throughout these investigations, together with the fact that AhR is highly evolutionarily conserved across a variety of animal species, ${ }^{24)}$ it has been suggested that AhR is involved in xenobiotic-independent and physiological functions. ${ }^{25)-27)}$ Furthermore, because AhR and Arnt (AhR nuclear translocator) are expressed during early mouse embryogenesis, ${ }^{28)}$ it has been proposed that AhR also plays a role in development. In addition, AhR has recently been reported to be involved in cell proliferation, apoptosis, adipose differentiation, tumor suppressor function, immune cell differentiation and reproductive function. ${ }^{29-31)}$ Consistent with these physiological roles of $\mathrm{AhR}$, it has been reported that $\mathrm{AhR}$ can be activated in response to cell density, even in the absence of obvious exogenous ligands, ${ }^{25), 32)}$ and in the presence of a variety of natural chemicals, such as lipoxin A4, prostaglandin G2 (PGG2), bilirubin and tryptophan derivatives including FICZ (6-formylindolo-[3,2-b]carbazole) and indirubin. ${ }^{33)}$ Moreover, the characterization of $\mathrm{AhR}$ homologues in invertebrates has indicated that these proteins are also involved in development, despite the fact that they cannot apparently bind xenobiotics. ${ }^{34)}$ Elucidation of the endogenous functions of AhR will provide deeper insight into the toxicological and adverse effects caused by xenobiotics, because these deleterious effects can be understood as a consequence of the unscheduled activation of AhR. In this short review, we will summarize and discuss our current knowledge on the regulatory mechanisms of $\mathrm{AhR}$ and its physiological functions in tumor suppression, reproduction, and immunity.

\section{Activation of AhR}

It is well established that $\mathrm{AhR}$ exists in a latent state in a complex with HSP90, immunophilin-like XAP2 (also named ARA9 or AIP), and p23 in the cytoplasm $^{17)}$ (Fig. 1A). HSP90 binding is essential to retain $\mathrm{AhR}$ in the cytoplasm and this interaction is thought to mask the nuclear localization signal (NLS) of AhR. Overexpression of XAP2 increases the amount of AhR in the cytosol. Furthermore, the LxxLL motif ( $\mathrm{x}$ is any amino acid) in the middle of the AhR molecule, which was found to mediate protein-protein interactions between transcriptional cofactors and nuclear receptors, is also involved in the cytoplasmic retention of AhR through proteinprotein interactions. ${ }^{25), 35)-37)}$ The roles of the three components (HSP90, XAP2 and p23) in the HSP90 complex are described in detail in a recent review. ${ }^{38)}$ Upon ligand binding, HSP90-bound AhR translocates into the nucleus and then dissociates from the HSP90 complex in the face of and binds to Arnt. ${ }^{17)}$ In in vitro experiments, HSP90 is released from the ligand-bound AhR when incubated with nuclear extracts from mouse Hepa-1 cells containing Arnt, but not from Arnt-deficient mutant cells, suggesting that Arnt stimulates the ligand-induced displacement of HSP90 from AhR. ${ }^{39)}$

It is well established that many synthetic PAHs and halogenated PAHs (HAHs) activate the AhR signal pathway. Although absolute planarity is not required for receptor binding, many agonists are planar compounds, and coplanarity is one of the most influential factors that affect the affinity of AhR for 
A

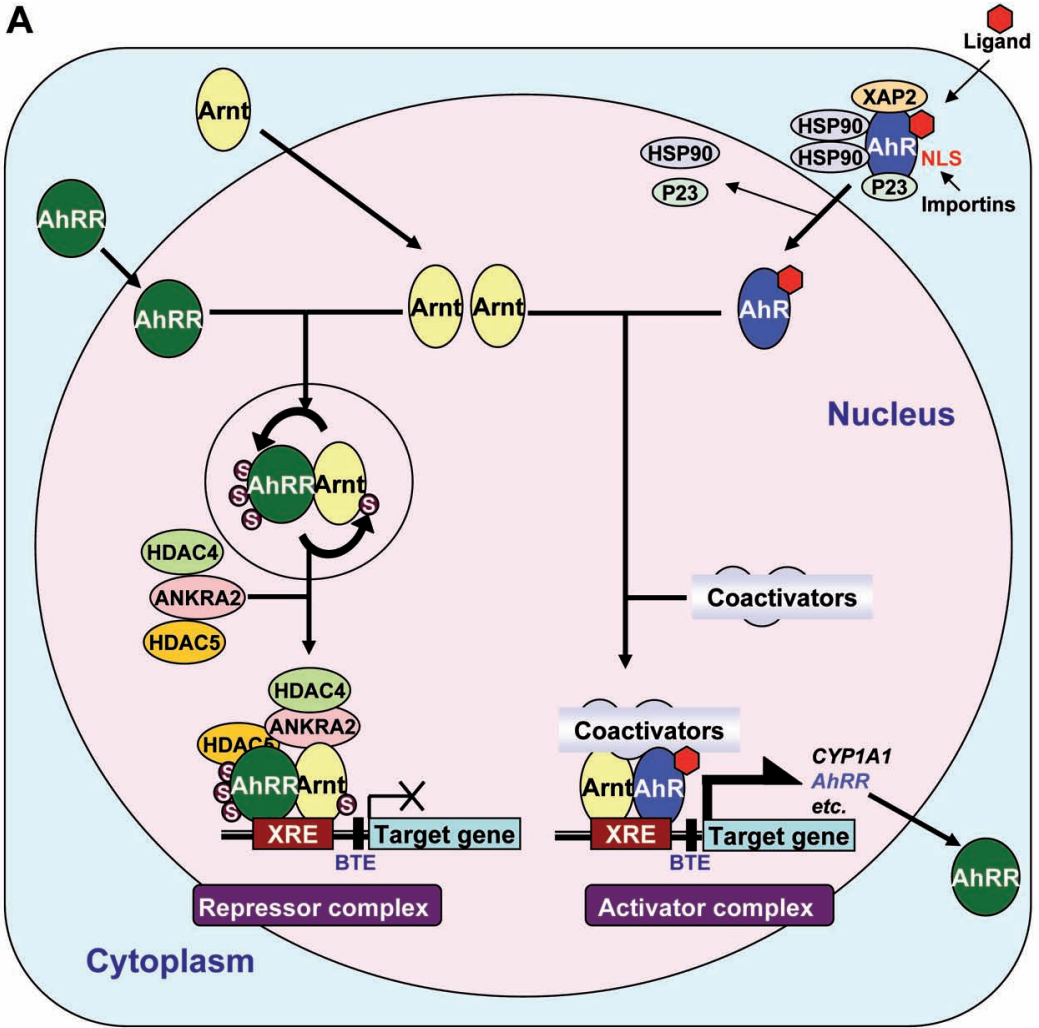

B

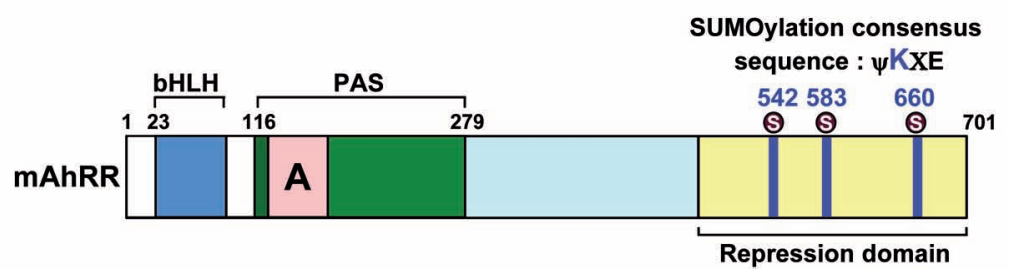

Fig. 1. A, A schematic model for the transcriptional regulation of the AhR/Arnt activator complex and the AhRR/Arnt repressor complex. Unmodified Arnt forms a heterodimer with AhR and recruits coactivators, such as CBP/p300, to form the transcriptional activator complex. Meanwhile, Arnt forms a heterodimer with AhRR, which significantly enhances the SUMOylation of both proteins. SUMOylated AhRR recruits corepressors ANKRA2, HDAC4, and HDAC5 to form the transcriptional repressor complex. B, A schematic representation of the full-length 701-amino acid mouse AhRR. The characterized domains represented are the basic helix-loop-helix (bHLH), Per-Arnt-Sim (PAS), and repression domain. Three putative SUMOylation sites are located within the repression domain, with the target lysine residues indicated.

XRE: Xenobiotic Responsive Element; BTE: Basic Transcription Element; a GC box sequence

its ligand. ${ }^{40)}$ A recent review assessed and discussed these xenobiotic ligands. ${ }^{40), 41)}$ In association with the increasing interest in the intrinsic functions of $\mathrm{AhR}$, natural or endogenous AhR ligands have been drawing much attention. These compounds contain tryptophan derivatives such as FICZ, IAA (indole-3-acetic acid), tryptamin, indirubin, ICZ (indolo[3,2-b]carbazole) and DIM (3,3'-diindolylmethane), prostaglan- dins such as PGG2 and lipoxin A4, and heme metabolites including bilirubin and biliruverdin. ${ }^{33), 40), 41)}$ However, it is quite difficult to determine which of these natural ligands are physiological cognate ligands or casual ones.

In contrast, several recent studies have reported ligand-independent AhR activation. When cells such as Hepa-1 and 10T1/2 fibroblasts are cultured in sus- 
pension, AhR translocates into the nucleus and induces CYP1A1 and 1B1 mRNA synthesis, even in the absence of an exogenous ligand.42),43) In addition, prevention of cell-cell interactions in the keratinocyte cell line, HaCaT, by growing the cells at a low cell density or in $\mathrm{Ca}^{2+}$-deficient conditions induces the nuclear accumulation of AhR, resulting in the activation of a reporter gene driven by XRE sequences. ${ }^{32)}$ It has been recently reported that addition of second messenger cAMP, an endogenous mediator of hormone and neurotransmitter signaling, to the culture medium activates and translocates $\mathrm{AhR}$ into the nucleus, leading to its nuclear accumulation. ${ }^{44)}$ The nuclear accumulation of $\mathrm{AhR}$ has been reported to be caused by phosphorylation of Ser 68 within the nuclear export sequence (NES). An analysis of specific kinase inhibitors suggests that this phosphorylation is catalyzed by p38MAPK. ${ }^{32)}$ An increasing number of reports have indicated that phosphorylation regulates the functions of $\mathrm{AhR}$ in physiological signaling pathways, such as cell proliferation and xenobiotic signal transduction. ${ }^{45)}$

Omeprazole is known to induce CYP1A1 in an AhR-dependent manner without directly binding to AhR. ${ }^{46), 47)}$ It is reported that tyrphosphins AG17 and AG879 tyrosine kinase inhibitors, selectively inhibited omeprazole-mediated induction of CYP1A1, while no inhibitory effect was observed on the TCDD-dependent induction of CYP1A1. Further, a Tyr320Phe mutation in AhR abolished omeprazole-dependent AhR signaling, but minimally affected TCDD-induced activation of CYP1A1 expression. These results suggest that Tyr320 is a phosphorylation site in AhR that is activated by omeprazole in a ligand-independent manner through a signal transduction pathway that involves protein tyrosine kinases. ${ }^{48)}$ Therefore, this signal pathway is independent of the AhR pathway that is induced by high affinity ligand binding. Although the detailed mechanisms are still unknown, it is highly likely that AhR is activated by phosphorylation in a ligand-independent manner.

\section{Transcriptional activation by AhR}

In the nucleus, the translocated $\mathrm{AhR}$ dissociates from the HSP90 complex and then heterodimerizes with Arnt, another bHLH-PAS protein, and the newly formed AhR/Arnt heterodimer binds XRE sequences in the promoters of target genes ${ }^{17)}$ (Fig. 1A). The mechanisms of AhR transcriptional enhance- ment are best characterized for the CYP1A1 gene. ${ }^{49)}$ In the approximately $2 \mathrm{~kb}$ sequence upstream of the CYP1A1 gene, a cluster of XREs (5'-TNGCGTG-3') functions as an enhancer ${ }^{11), 13)}$ and a basic transcription element (BTE) of a GC box sequence located immediately upstream of the gene is also required for high CYP1A1 expression levels50) (Fig. 1A). SP1 binds the BTE, while the AhR/Arnt heterodimer binds the XRE sequence. ${ }^{50), 51)}$ The binding of these two transcription factors to their cognate enhancer sequences is synergistic; the binding of one of the transcription factors to its cognate sequence facilitates the binding of the other to its cognate sequence. It is also interesting that the sequences of the XRE and GC box are frequently arranged in the promoters of AhR-target genes. ${ }^{52)}$

It has been reported that binding of the AhR/ Arnt heterodimer to XREs induces chromatin remodeling, resulting in DNase hyper-sensitivity within 300 bp upstream of the transcription start site of the target genes. ${ }^{49), 53)}$ Furthermore, studies have shown that when it binds to the XRE sequence, the AhR/ Arnt heterodimer recruits CBP/p300, SRC1, NCoA2/ GRIP1/TIF2, p/CIP and other coactivators including RIP140, components of ATP-dependent chromatin remodeling complexes such as BRG-1, p-TEF $\beta$ and RNA elongation factors. ${ }^{54), 55)}$ In addition, the TRAP/DRIP/ ARC/Mediator complex is recruited to the CYP1A1 promoter to activate target gene expression in response to xenobiotic stress. ${ }^{54), 56)}$ Although multiple components are reported to form the transcription complex on the AhR/Arnt heterodimer, the interactions and order of the complex formation have only begun to be partially elucidated, and the current state of these studies has been recently reviewed in detail. ${ }^{38)}$

\section{Negative regulation of AhR activity}

AhR signaling can be downregulated by at least two independent mechanisms, proteasomal degradation of $\mathrm{AhR}$ and competitive inhibition of AhR by the $A h R$ repressor $(A h R R)$, which is induced by activated AhR (Fig 2). It has been reported that the AhR protein is rapidly depleted in vitro, following exposure to AhR ligands, most likely after target gene activation. ${ }^{57-59)}$ This AhR degradation has been observed in rat smooth muscle A7, murine Hepa-1 and human HepG2 cells and was blocked by treating with the proteasome inhibitor MG-132. Since this degradation was inhibited by leptomycin B (LMB), a nuclear export inhibitor, it is likely that $\mathrm{AhR}$ degradation 


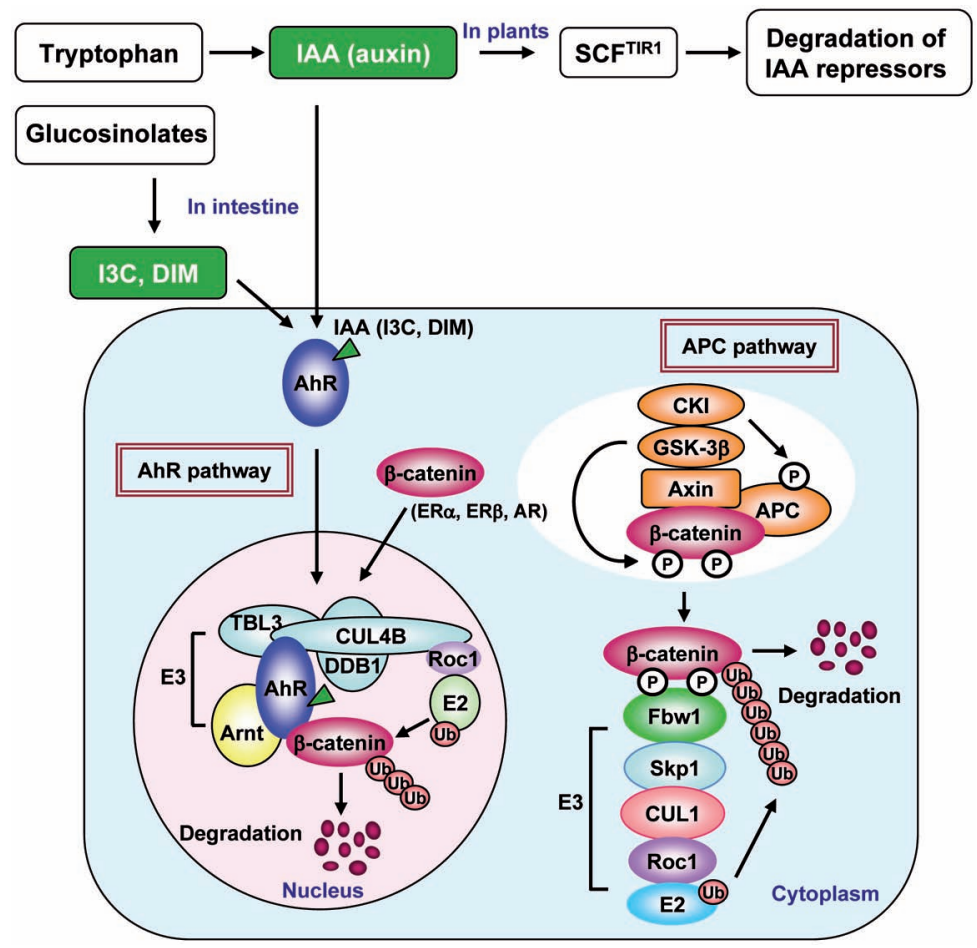

Fig. 2. A schematic model of the AhR- and APC-dependent $\beta$-catenin degradation pathways. Ligand-activated AhR translocates into the nucleus where it forms a ubiquitylation complex containing CUL4B and Arnt, whereas the APC-dependentpathway functions in the cytoplasm. This function of AhR is activated by both xenobiotics and natural AhR ligands, such as indole derivatives (IAA, I3C, DIM. etc.) that are converted from dietary tryptophan and glucosinolates by intestinal microbes.

occurs in the cytoplasm. ${ }^{57), 58)}$ However, AhR degradation was also shown to occur in the nucleus, and this degradation did not require nuclear export. The ubiquitin ligase protein, C-terminal hsp70-interacting protein (CHIP), has also been implicated in the nuclear degradation of AhR. ${ }^{59)}$

Recently, we have reported that when AhR is ligand-activated and translocates into nucleus, it forms an E3 ubiquitin ligase complex with CUL4B (cullin4B), DDB1 (damaged-DNA-binding 1), TBL3 (transducin $\beta$-like 3 ) and Rbx1 (ring box protein 1)/ Roc1 and catalyzes the ubiquitylation of sex steroid hormone receptors, ER $\alpha, \mathrm{ER} \beta, \mathrm{AR}$ (androgen receptor) and AhR itself. ${ }^{60)}$ Very recently, we have found that this AhR E3 ubiquitin ligase ubiquitylates $\beta$ catenin and functions as a tumor suppressor in the colon, as will be described later in detail. ${ }^{61)}$ In this complex, CUL4B and AhR are thought to serve as the scaffold and substrate recognition component, respectively. The functional relationship between the two ubiquitylation pathways in the nucleus remains to be investigated.
Like other bHLH-PAS signaling pathways such as HIFs, ${ }^{62)}$ we have revealed that the AhR pathway is also downregulated through feedback inhibition. AhRR was originally found as a TCDD-induced protein and subsequently shown to inhibit AhR transcriptional activity. ${ }^{63)}$ The $A h R R$ promoter has a functional XRE sequence, and gene expression is enhanced upon ligand activation of AhR, resulting in the inhibition of AhR signaling activity. ${ }^{63), 64)}$ AhRR contains a bHLH-PASA region that is structurally similar with $\mathrm{AhR}$, followed by a C-terminal transcription repression domain (Fig. 1B). AhRR also forms a heterodimer with Arnt ${ }^{63)}$ (Fig. 1A). Since AhRR has both NLS and NES sequences and does not require an agonist to dimerize with Arnt, newly synthesized AhRR translocates into nucleus and forms a heterodimer with Arnt. This heterodimer binds competitively to the XRE sequence with the AhR/Arnt heterodimer, and subsequently recruits co-repressors such as ANKRA2, HDAC4 and HDAC5. ${ }^{65)}$ The Cterminal repression domain of AhRR has three SUMOylation sites that are conserved across verte- 
brate species (Fig. 1A), and all three sites must be SUMOylated for full suppressive activity. ${ }^{66)} \mathrm{In} \mathrm{MEF}$ cells that do not express CYP1A1, AhRR/Arnt was shown to function as a repressor by ChIP (chromatin immunoprecipitation) analysis. ${ }^{65)}$ It has been recently reported that AhRR functions as a putative tumor suppressor ${ }^{67)}$ and that susceptibility to endometriosis and male infertility are associated with genetic polymorphisms in the AhRR gene. ${ }^{68)-70)}$ The two independent mechanisms of downregulating the AhR signaling pathways implicate that there is evolutionary selection against over-activation of AhR.

\section{The E3 ubiquitylation and tumor suppressor functions of AhR}

It is well known that AhR ligands negatively or positively regulate the estrogen receptor (ER) activity. ${ }^{71), 72)}$ The loss of the transactivation potential of ER in the presence of TCDD is reportedly due to a sharp decrease in its ability to bind to the estrogen response element (ERE). AhR ligand-induced degradation and functional repression of sex steroid hormone receptors were attenuated by treating with the proteasome inhibitor, MG-132, resulting in polyubiquitylation of the ER, as determined by polyacrylamide gel electrophoresis. ${ }^{73}$ ) Consistent with this observation, we revealed that polyubiquitylation of $\mathrm{ER} \alpha$ and even AhR was promoted by AhR ligands in AhR immunoprecipitated complexes, when ubiquitin E1 and E2 ligases were supplemented in vitro. ${ }^{60)}$ The AhR-containing complexes were isolated from 3MC-treated HeLa cells expressing Flagtagged AhR by anti-Flag antibody. Glycerol gradient centrifugation of the isolated AhR-containing complexes revealed five major 3MC-dependent complexes. While two of these complexes contained well known coactivators TRAP220/DRIP205/Med220 and p300 as transcription components, one complex was composed of the ubiquitin ligase core components, CUL4B, DDB1 and Rbx (Roc), along with subunits of proteasomal 19S regulatory particle (19SRP), Arnt and TBL3. ${ }^{60)}$

When individual recombinant AhR, CUL4B, DDB1, and Rbx1 proteins from Sf9 cells were incubated together, the complex formation was dependent on $3 \mathrm{MC}$, and the complex exhibited ubiquitin ligase activity for the ER in vitro when supplemented with E1 and E2 ligases. We revealed that within this AhR ubiquitinylation complex, AhR recognizes and binds both substrates and CUL4B as a scaffold to form the ubiquitinylation complex. ER $\alpha, \operatorname{ER} \beta$, and
$\mathrm{AR}$ are found to be substrates for the CUL4B/AhR complex (Fig. 2). It was previously reported that human $\mathrm{ER} \alpha(\mathrm{hER} \alpha)$ degradation is accelerated upon E2 ligand binding or phosphorylation of Ser112, whereas a partial agonist, tamoxifen, stabilizes $\mathrm{ER} \alpha .^{74), 75)}$ In contrast, we have shown the 3MC-activated AhR to induce the ubiquitylation and subsequent degradation of tamoxifen-bound ER $\alpha$ and the ER $\alpha$ S118A mutant. ${ }^{60)}$ Furthermore, AhR was dispensable for E2dependent ER $\alpha$ degradation. These results indicate that the CUL4B/AhR complex may act independently of the innate protein degradation system(s) for ER $\alpha{ }^{76)}$

Most recently, our result has revealed that the CUL4B/AhR complex ubiquitylates and degrades $\beta$ catenin in intestinal epitherial cells and functions as a tumor suppressor in the intestine. ${ }^{61)} \mathrm{AhR}$-deficient mice were found to frequently develop colonic tumors, mostly in the cecum at approximately 10 weeks of age. ${ }^{61)}$ It is well known that the APC ubiquitylation system in the colon functions as a tumor suppressor to regulate the $\beta$-catenin concentration in the intestine $^{77)}$ (Fig. 2). Because 3MC- or $\beta$-naphthoflavone $(\beta \mathrm{NF})$-induced $\mathrm{AhR}$ activation was associated with markedly decreased endogenous $\beta$-catenin levels in DLD, SW480, or HCT116 cells derived from colon cancers, which are known to stabilize $\beta$-catenin because of mutations in APC or the $\beta$-catenin gene, ${ }^{78)}$ it has been suggested that APC- and AhR-containing ubiquitylation complexes function independently. Furthermore, we have revealed that siRNA-mediated knockdown of AhR or CUL4B, but not APC reversed repression of the $\beta$-catenin transcription activity on TCF/LEF that was induced by the AhR ligands, $3 \mathrm{MC}, \beta \mathrm{NF}$, and IAA in an in vitro reporter gene assay. ${ }^{61)}$ These results confirm that $\mathrm{AhR}$ is involved in previously undescribed ligand-dependent mechanisms of proteasome-mediated $\beta$-catenin degradation that are distinct from the canonical APCdependent pathway.

We investigated the functional association between the APC- and AhR-dependent pathways of $\beta$ catenin degradation in intestinal tumor development by generating the mice with compound mutations in the $A P C$ and $A h R$ genes. These $A P C^{\mathrm{Min} /+} \cdot A h R^{+/-}$ and $A P C^{M i n /+} \cdot A h R^{-/-}$mice had greatly accelerated intestinal carcinogenesis compared with the parental mice that had single mutations in the respective genes. These results provide further support that $\mathrm{AhR}$ and APC have a cooperative tumor suppression function in intestinal epitherial cells. In connection 
with these observations, the compound mutant mice had much higher $\beta$-catenin expression in their intestines than the respective parental mutant mice. These results indicate that the AhR ubiquitylation complex functions as tumor suppressor independently of and parallel to the canonical APC-dependent pathway. While the APC system is cytoplasmic, AhR which is activated by ligand-binding is translocated into the nucleus where it forms a ubiquitylation complex. Therefore, these two ubiquitylation complexes are considered to function in different cellular compartments (Fig. 2).

It has been reported that natural AhR ligands derived from indole, such as IAA, I3C (indole-3carbinol) and DIM, are generated through the conversion of dietary tryptophan and glucosinolates by commensal intestinal microbes and exert chemopreventive effects on colorectal cancers in humans. ${ }^{79)-81)}$ Remarkably, $A P C^{M i n /+}$ or $A P C^{M i n /+} \cdot A h R^{+/-}$mice, which develop multiple intestinal tumors on a normal diet, show reduced levels of carcinogenesis in the intestine when fed an I3C- or DIM-containing diet immediately after weaning at 3 or 4 weeks of age. We conclude that these chemopreventive effects of I3C and DIM are mediated by AhR, because no tumor suppressive effect was observed in $A h R^{-/-}$mice. ${ }^{61)}$ Since AhR shows a broad ligand specificity, the ability to bind to AhR should provide an effective screening method for the most suitable chemopreventive chemicals against carcinogenesis.

\section{Novel physiological functions of AhR}

AhR in reproduction. Previous studies have shown that the lack of $A h R$ gene expression in $A h R$ null mice and the unscheduled activation of the AhR signaling pathway by HAHs result in adverse phenotypes in female reproduction organs and lead to impaired reproduction.82) Together with the fact that AhR is ubiquitously expressed in female genital organs, these results indicate that AhR plays an important physiological role in female reproduction. Female AhR-null mice exhibit defects in multiple reproductive aspects, such as conception, litter size and pup survival. ${ }^{83), 84)}$ These defective functions are reflected in the decreased number of ovulated oocytes and disordered estrous cycle. ${ }^{84)}$ The estrous cycle and oocyte development in the ovary are regulated by the coordinated actions of the anterior pituitary hormones, LH and FSH, and ovarian steroid hormones including androgen, estrogen and progestin. ${ }^{82), 85)}$ During the normal estrous cycle, hormonal signals induce the maturation of granulosa cells surrounding the oocyte, trigger follicle rupture and lead to the subsequent release of the oocyte into the fallopian tube. ${ }^{82)}$ Our result has revealed that while the number and morphology of immature, preovulatory follicles were similar between wild-type and $A h R$-null females, ${ }^{82), 84), 86)}$ the numbers of mature follicles and corpora lutea (CL) were remarkably reduced in the super-ovulation process. ${ }^{84}$ Several lines of evidence including ours showed that serum LH and FSH levels were similar between $A h R$-null and wild-type mice at all stages of the mouse estrous cycle, but that reduced estradiol synthesis within the ovary caused defective reproduction in $A h R$-null female mice. ${ }^{84), 86), 87)}$

It is known that estrogen is synthesized from cholesterol in the ovary and, therefore, that all the enzymes involved in estradiol synthesis are present in the ovary. ${ }^{88), 89)}$ Specifically, FSH secreted from the anterior pituitary binds to the FSH receptor (FSHR) to stimulate P450 aromatase (CYP19) expression, the rate-limiting enzyme in estrogen synthesis that converts testosterone to estradiol, with a peak of expression in the proestrous stage in granulosa cells. ${ }^{90)}$ In contrast, other steroidogenic enzymes such as $\mathrm{P} 450_{\mathrm{SCC}}(\mathrm{CYP} 11 \mathrm{~A}), \mathrm{P} 450$ 17a-hydroxylase

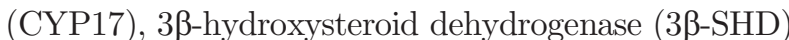
and $17 \beta$-hydroxysteroid dehydrogenase (17ß-SHD) are constitutively expressed.90) We have revealed that although the expression of steroidogenic enzymes other than CYP19 was essentially unchanged between $A h R$-deficient and wild-type mice, CYP19 expression was not enhanced in the proestrous stage in AhR-deficient mice, leading to markedly reduced ovarian estradiol concentrations compared to wildtype mice. ${ }^{84), 91)}$ We have also found that the female reproductive defects in $C Y P 19 \mathrm{KO}$ and $E R \mathrm{KO}$ mice are similar to those in $A h R \mathrm{KO}$ mice, albeit with more severe phenotypes. ${ }^{84), 92), 93)}$ The phenotypic similarities in defective reproduction between AhR KO, CYP19 KO and ER KO mice provide further evidence that $A h R \mathrm{KO}$ mice have deficits in estrogen production or function.

The AhR and Ad4BP/SF-1 binding sites in the promoter of the $C Y P 19$ gene are conserved in several vertebrates, including many kinds of fish, humans, and mice, suggesting an evolutionarily conserved role for the interactions between these binding sites and their cognate transcription factors in ovarian physiology. ${ }^{84)}$ Furthermore, reporter gene assay re- 
vealed that $\mathrm{AhR}$ and $\mathrm{Ad} 4 \mathrm{BP}$ synergistically activated the promoter of the CYP19 gene. The interaction between AhR and Ad4BP transcription factors was confirmed by ChIP analysis. ${ }^{84)}$ In addition, interestingly, we have found that in a super-ovulation experiment, the expression of both AhRR and CYP19 was similarly regulated in an AhR-dependent manner, while the CYP19 gene peaked earlier than AhRR, which was upregulated when CYP19 expression began to decline. It is possible that $\mathrm{AhR}$ and AhRR regulate the ovarian biological clock by governing the estrous cycle. Several lines of evidence indicate that $\mathrm{AhR}$ plays physiological roles in reproduction beyond the estrous cycle. ${ }^{26), 82)}$ During the peri-implantation period, AhR is abundantly expressed in the uterine vasculature and developing tissues between the embryo and mother. ${ }^{94)}$ In addition to reduced fertility, $A h R \mathrm{KO}$ females have difficulty in raising their pups to the weaning age, and these pups have a significantly lower survival rate than pups raised by heterozygous females, ${ }^{83)}$ a phenotype that may be related to impaired development of the mammary glands. ${ }^{95}$ )

AhR in immunity. There is strong evidence that AhR plays significant roles in the immune system. ${ }^{96)}$ Exposure to TCDD leads to severe thymic involution and profound suppression of both the humoral and cellular immune responses, resulting in increased susceptibility to infection. In addition, $A h R \mathrm{KO}$ mice are defective in $\mathrm{T}$ cell differentiation and are more susceptible to bacterial infection. ${ }^{97,98)}$ Most of the cells that participate in immune responses constitutively or inducibly express $\mathrm{AhR}$ at substantial levels. Many genes that participate in the immune response, such as all 9 TLRs, IL-1Rs, IL-6, IL-18, etc., have XRE sequences in their promoters, although it is mostly unknown whether $\mathrm{AhR}$ is directly involved in their expression. ${ }^{97), 98)}$

Although the mechanism has not been investigated in detail, greatly enhanced TNF $\alpha$ and IL- $1 \beta$ production from macrophages rendered the AhR wild-type mice treated with TCDD much more susceptible to LPS-induced septic shock. ${ }^{99), 100)}$ In contrast, we have observed that the $A h R \mathrm{KO}$ mice were also hypersensitive to LPS-induced lethal shock compared to wild-type mice. ${ }^{101), 102)}$ Upon LPS treatment, the serum levels of IL-6, TNF $\alpha$ and IL-1 $\beta$ were significantly higher in $A h R \mathrm{KO}$ mice than wildtype mice. Consistent with these in vivo results, we have revealed that peritoneal macrophages isolated from $A h R \mathrm{KO}$ mice induced much higher IL-6 secretion in response to LPS treatment than those from wild-type mice. Furthermore, transfecting the $A h R$ $\mathrm{KO}$ macrophages with an AhR expression plasmid suppressed this enhanced IL-6 expression upon LPS treatment. ${ }^{101)}$ These results indicate that AhR suppresses the expression of inflammatory cytokines in response to LPS. Interestingly, we have revealed that peritoneal macrophages markedly enhanced AhR expression in response to LPS treatment. ${ }^{102)}$ It has been suggested that AhR plays a suppressive role by forming a complex with Stat1 and NF- $\mathrm{KB}$ in macrophages, leading to the inhibition of the promoter activity of the $I L-6$ gene. ${ }^{101)}$

In the case of IL- $1 \beta$ secretion, we have observed that AhR activates the expression of Pai- $2,{ }^{102}$ an inhibitor of caspase- 1 activation that functions in the process of IL- $1 \beta$ secretion. ${ }^{103)}$ AhR ligands markedly enhanced Pai-2 expression in wild-type macrophages and transduction of Pai-2 into AhR KO macrophages restored suppression of IL-1 $\beta$ secretion. ${ }^{102)}$ Activated AhR together with NF- $\mathrm{KB}$, but not with Arnt, directly enhanced the expression of Pai-2 gene by binding to its promoter. ${ }^{102)}$ It is reported that experiments with the human macrophage cell line U937 showed that TCDD induces the expression of IL-8, $\mathrm{Bcl}$ (B-cell chemoattractant) and Baff (B-cell activating factor). ${ }^{104)}$ These increases in gene expression were associated with the binding of the AhR-Rel complex to a novel NF- $\kappa B$ binding site in the promoters of these genes, and this process did not require Arnt, suggesting a new mechanism of transcriptional regulation that involves TCDD-activated AhR. ${ }^{104)}$

There have been several lines of evidence indicating that AhR ligands stimulate naïve $\mathrm{T}$ cells (Th0) to differentiate into helper T-cell subsets that suppress or accelerate the immune responses by modulating effector $\mathrm{T}$ cell proliferation and cytokine secretion. ${ }^{97), 98)}$ Previous studies have shown that the profound suppression of an acute graft versus host $(\mathrm{GVH})$ response in TCDD-treated mice was associated with the generation of donor-derived $\mathrm{CD} 4^{+}$ $\mathrm{CD} 25^{+} \mathrm{T}_{\text {reg }}$ cells that were dependent upon AhR activation. ${ }^{105), 106)} \mathrm{VAF}$ is a low molecular weight compound that activates AhR. In vivo VAG539 treatment increased the frequency of splenic $\mathrm{CD} 4^{+}$ $\mathrm{T}$ cells expressing CD25 and FoxP3 and induced pancreatic islet allograft tolerance through direct as well as DC-mediated induction of $\mathrm{T}_{\text {reg }}$ cells. $\left.{ }^{106}\right) \mathrm{We}$ have reported that another AhR ligand, M50354, 
exerts an anti-allergic effect in an AhR-dependent manner both in vitro and in vivo. ${ }^{107)}$ TCDD-induced $A h R$ activation was found to induce functional $T_{\text {reg }}$ cells that suppressed experimental autoimmune encephalomyelitis (EAE). ${ }^{108)}$ Interestingly, incubation of Th0 cells under $\mathrm{T}_{\text {reg }}$ polarizing conditions containing TGF $\beta_{1}$ along with CD3 and CD28 antibodies induced AhR expression. Furthermore, the promoter of the FoxP3 gene, a transcription factor thought to play a master role in $\mathrm{T}_{\text {reg }}$ differentiation, contains functional XRE sequences that are responsible for inducing this gene in response to activated AhR. In addition, ChIP analysis revealed that $\mathrm{AhR}$ was recruited to the FoxP3 promoter. ${ }^{108)}$

It has been recently reported that AhR plays an essential role in the development of IL-17-producing $\mathrm{T}$ helper cells (Th17), a new subset of $\mathrm{CD} 4^{+} \mathrm{T}$ cells thought to be involved in autoimmunity and the clearance of infectious agents. ${ }^{109), 110)}$ Th17 cell development is reciprocally related to the generation of $\mathrm{T}_{\text {reg }}$ cells. While TGF $\beta_{1}$ induces the differentiation of $\mathrm{T}_{\text {reg }}$ cells, TGF $\beta_{1}$ in combination with IL-6 boosts the differentiation of Th17 cells from naïve $\mathrm{T}$ cells under $\mathrm{T}$ cell differentiation conditions. The presence of an AhR ligand, FICZ, further enhances Th17 differentiation under Th17-polarizing conditions. AhR expression is remarkably accelerated in induced Th17 cells, with approximately 10-fold higher expression than that in induced $\mathrm{T}_{\text {reg }}$ cells. ${ }^{109), 110)}$ Consistent with these observations, we have revealed that Th17 cell differentiation is severely impaired in $A h R \mathrm{KO}$ Th0 mice, despite normal expression of ROR $\alpha$ and $\operatorname{ROR} \gamma$, which are thought to be key players in Th17 cell differentiation. ${ }^{110)}$ FICZ treatment exacerbates EAE symptoms in wild-type mice compared to $A h R$ $\mathrm{KO}$ or untreated wild-type mice. ${ }^{109)}$ We have suggested that Th17 cell differentiation is stimulated by activated AhR through interactions with Stat1 and Stat5, which negatively regulate Th17 cell development. ${ }^{110)}$ Although the molecular mechanisms by which $\mathrm{AhR}$ regulates the helper $\mathrm{T}$ cell differentiation process are still unclear, these results indicate that $\mathrm{AhR}$ is centrally involved in regulating immune responses by modulating the $\mathrm{T}_{\mathrm{reg}} / \mathrm{Th} 17$ balance through its ligand in specific cytokine milieus. In addition, these data suggest that $\mathrm{AhR}$ is a unique target for therapeutic manipulation of immune responses.

\section{Conclusions}

The molecular mechanisms of the transcriptional activity of AhR have been elucidated in more detail, with particular emphasis on coactivators, mediators and chromatin remodeling factors. Furthermore, there is increasing evidence that $\mathrm{AhR}$ can be activated in a ligand-independent manner, although this mechanism needs to be further studied. The AhR signaling pathway can be precisely regulated by a feedback inhibitory mechanism in which AhRR, whose expression is enhanced by activated AhR, represses the transcription activity of AhR via SUMOylation and the subsequent recruitment of corepressors. In addition to the role of AhR in toxicology and pharmacology, recent evidence indicates that AhR is also involved in reproduction and innate immunity. One of the major contributions of $\mathrm{AhR}$ is to protect against invading agents ranging from various small molecule chemicals to microorganisms. With the novel finding that $\mathrm{AhR}$ is involved in ubiquitylation, our knowledge on the function of AhR further extends into tumor suppression and the regulation of steroid hormones.

\section{Acknowledgements}

Our work was funded in part by Core Research for Evolutional Science and Technology (CREST), and Solution Oriented Research for Science and Technology (SORST) from Japan Science and Technology Agency (JST), 4-1-8 Honcho, Kawaguchi 3320021, Japan and by a grant for Scientific Research from Ministry of Health, Labor and Welfare, Japan.

\section{References}

1) Conney, A.H. (1982) Induction of microsomal enzymes by foreign chemicals and carcinogenesis by polycyclic aromatic hydrocarbons: G.H.A. Clowes Memorial Lecture. Cancer Res. 42, 4875-4917.

2) Kato, R. (1961) Modification of the toxicity of strychnine and octomethylpyrophosphoramide (OMPA) induced pretreatment with phenaglycodol and thiopental. Arzneim.-Forsch. 11, 797-798.

3) Remmer, H. (1959) Die Beschleunigung der evipanoxydation und der demethylierung von methylaminoantipyrin durch barbiturate. Arch. Exp. Pathol. Pharmacol. 237, 296-307.

4) Conney, A.H. and Burn, J.J. (1959) Stimulatory effect of foreign compounds on ascorbic acid biosynthesis and drug-metabolizing enzymes. Nature 184, 363-364.

5) Omura, T. and Sato, R. (1962) A new cytochrome in liver microsomes. J. Biol. Chem. 237, 1375-1376.

6) Cooper, D.Y., Levin, S., Narasimhulu, S., Rosenthal, O. and Estabrook, R. (1965) Photochemical action spectrum of the terminal oxidase of mixed function oxidase systems. Science 147, 400-402. 
7) Poland, A., Glover, E. and Kende, A.S. (1976) Stereospecific, high affinity binding of 2,3,7,8-tetrachlorodibenzo- $p$-dioxin by hepatic cytosol. Evidence that the binding species is receptor for induction of aryl hydrocarbon hydroxylase. J. Biol. Chem. 251, 4936-4946.

8) Greenlee, W.F. and Poland, A. (1979) Nuclear uptake of 2,3,7.8-tetrachlorodibenzo-p-dioxin in C57BL/6J and DBA/2J mice. Role of hepatic cytosol receptor protein. J. Biol. Chem. 254, 9814-9821.

9) Fujii-Kuriyama, Y., Mizukami, Y., Kawajiri, K., Sogawa, K. and Muramatsu, M. (1982) Primary structure of a cytochrome P-450: Coding nucleotide sequence of phenbarbital-inducible cytochrome P-450 cDNA. Proc. Natl. Acad. Sci. USA 79, 2793-2797.

10) Sogawa, K., Gotoh, O., Kawajiri, K. and FujiiKuriyama, Y. (1984) Distinct organzation of methylcholanthrene- and phenobarbital-inducible cytochrome P-450 genes in rat. Proc. Natl. Acad. Sci. USA 81, 5066-5070.

11) Fujisawa-Sehara, A., Sogawa, K., Yamane, M. and Fujii-Kuriyama, Y. (1987) Characterization of xenobiotic responsive elements upstream from drugmetabolizing cytochrome P-450c gene: a similarity to glucocorticoid regulatory elements. Nucleic Acid Res. 15, 4179-4191.

12) Fujisawa-Sehara, A. Yamane, M. and Fujii-Kuriyama, Y. (1988) A DNA-binding factor specific for xenobiotic responsive elements of $P-450 \mathrm{c}$ gene exists as a cryptic form in cytoplasm: its possible translocation to nucleus. Proc. Natl. Acad. Sci. USA 85, 5859-5863.

13) Denison, M.S., Fisher, J.M. and Whitlock Jr., J.P. (1988) The DNA recognition site for the dioxin-Ah receptor complex. Nucleotide sequence and functional analysis. J. Biol. Chem. 263, 17221-17224.

14) Bradfield, C.A., Glover, E. and Poland, A. (1991) Purification and N-terminal amino acid of the Ah receptor from the C57BL/6J mouse. Mol. Pharm. 88, 306-312.

15) Ema, M., Sogawa, K., Watanabe, N., Chujoh, Y., Matsushita, N., Gotoh, O., Funae, Y. and FujiiKuriyama, Y. (1992) cDNA cloning and structure of mouse putative Ah receptor. Biochem. Biophys. Res. Commun. 184, 246-253.

16) Burbach, K.M., Poland, A. and Bradfield, C.A. (1992) Cloning of the Ah-receptor cDNA reveals a distinctive ligand-activated transcription factor. Proc. Natl. Acad. Sci. USA 89, 8185-8189.

17) Hankinson, O. (1995) The aryl hydrocarbon complex. Annu. Rev. Pharmacol. Toxicol. 35, 307-340.

18) Okey, A.B. (2007) Aryl hyrocarbon receptor odyssay to the shores of toxicology: Deichmann lecture. International Congress of Toxicology-XI. Toxicol. Sci. 98, 5-38.

19) Fernandez-Salguero, P., Pinean, T., Hilbert, D.M., McPhail, T., Lee, S.S., Kimura, S., et al. (1995) Immune system impairment and hepatic fibrosis in mice lacking the dioxin-binding $\mathrm{Ah}$ receptor. Science 268, 722-726.

20) Schmidt, J.V., Su, G.H., Raddy, J.K., Simon, M.C. and Bradfield, C.A. (1996) Characterization of a murine Ahr null allele: Involvement of the the Ah receptor in hepatic growth and development. Proc. Natl. Acad. Sci. USA 93, 6731-6736.

21) Mimura, J., Yamashita, K., Nakamura, K., Morita, M., Takagi, T.N., Nakao, K. et al. (1997) Loss of teratogenic response to 2,3,7,8-tetrachlorodibezo- $p$ dioxin (TCDD) in mice lacking Ah (dioxin) receptor. Genes Cells 2, 645-654.

22) Mimura, J. and Fujii-Kuriyama, Y. (2003) Functioal role of $\mathrm{AhR}$ in the expression of toxic effects of TCDD. Biochem. Biophys. Acta 1619, 263-268.

23) Gonzalez, F.J. and Fernandez-Salguero, P. (1998) The aryl hydrocarbon receptor: Studies using the AHR-null mice. Drug Metab. Dispos. 26, 1194 1198.

24) Hahn, M.E. (2002) The aryl hydrocarbon receptor: Diversity and evolution. Chem. Biol. Interact. 141, 131-160.

25) Kawajiri, K. and Fujii-Kuriyama, Y. (2007) Cytochrome P450 gene regulation and physiological functions mediated by the aryl hydrocarbon receptor. Arch. Biochem. Biophys. 464, 207-212.

26) McMillan, B.J. and Bradfield, C.A. (2007) The aryl hydrocarbon receptor sans xenobiotics: endogenous function in genetic model system. Mol. Pharm. 72, 487-498.

27) Barouki, R., Coumoul, X. and Fernandez-Salguero, P.M. (2007) The aryl hydrocarbon receprtor, more than a xenobiotic-interacting protein. FEBS Lett. 581, 3608-3615.

28) Bryant, P.L., Clark, G.C., Prost, M.R. and Abott, B.O. (1997) Effects of TCDD on Ah receptor, Arnt, EGF, and TGF-alpha expression in embryonic mouse tract. Teratology 55, 326-337.

29) Puga, A., Ma, C. and Marlowe, J.L. (2009) The aryl hydrocarbon receptor cross-talks with multiple signal transduction pathway. Biochem. Pharmacol. 77, 713-722.

30) Hernandez-Ochoa, I., Karman, B.N. and Flaws, B.N (2009) The role of the aryl hydrocarbon receptor in the female reproduction system. Biochem. Pharmacol. 77, 547-559.

31) Kerkvliet, N.I. (2002) Recent advances in understanding the mechanisms of TCDD immunology. Int. Immunopharmacol. 2, 277-291.

32) Ikuta, T., Kobayashi, Y. and Kawajiri, K. (2004) Cell density regulates intracellular localization of aryl hydrocarbon receptor. J. Biol. Chem. 279, 19209-19216.

33) Nguyen, L.P. and Bradfield, C.A. (2008) The search for endogenous activator of the aryl hydrocarbon receptor. Chem. Biol. Toxicol. 21, 102-116.

34) Hahn, M.E. (2002) Aryl hydrocarbon receptor: Diversity and evolution. Chem. Biol. Interact. 141, 131-160.

35) Kashuba, E.V., Grading, K., Isaguliants, M., Szekely, L., Poellinger, L., Klein, G. and Kazlauskas, A. (2006) Regulation of transactivation function of the aryl hydrocarbon receptor. J. Biol. Chem. 281, 1215-1223. 
36) LaPres, J.J., Glover, E., Dunham, E.E. and Bradfield, C.A. (2000) ARA9 modifies agonist signaling through an increase in cytosolic aryl hydrocarbon receptor. J. Biol. Chem. 275, 6153-6159.

37) Ikuta, T., Watanabe, J. and Kawajiri, K. (2002) Characterization of the LXXLL motif in the aryl hydrocarbon receptor: Effects on subcellular localization and transcriptional activity. J. Biochem. 131, 79-85.

38) Beischlag, T.V., Morales J.L., Hollingsheed, B.D. and Perdew, G.H. (2008) The aryl hydrocarbon receptor complex and the control of gene expression. Crit. Rev. Eukaryot. Gene Expr. 18, 207-250.

39) Mcguire, J., Whitelaw, M.L., Pongratz, I., Gustaffson, J.A. and Poellinger, L. (1994) A cellular factor stimulates ligand-dependent release of hsp90 from the basic helix-loop-herix dioxin receptor. Mol. Cell. Biol. 14, 2438-2446.

40) Denison, M.S. and Nagy, S.R. (2003) Activation of the aryl hydrocarbon by by structurally diverse exogenous and endogenous chemicals. Annu. Rev. Pharmacol. Toxicol. 43, 309-334.

41) Ashida, H., Nishiumi, S. and Fukuda, I. (2008) An update on the dietary ligands of AhR. Expert Opin. Drug Metab. Toxicol. 4, 1429-1447.

42) Sadek, C.M. and Allen-Hoffmann, B.L. (1994) Suspension-mediated induction of Hepa 1c1c7 Cyp 1a1 expression is dependent on the Ah receptor signal transduction pathway. J. Biol. Chem. 269, 31503159.

43) Cho, Y.C., Zheng, W. and Jefcoat, C.R. (2004) Disruption of cell-cell contact maximally but transiently activates AhR-mediated transcription in 10T1/2 fibroblast. Toxicol. Appl. Pharmacol. 199, 220-238.

44) Oesch-Bartlomowicz, B., Huelster, A., Wiss, O., Antoniou-Lipfert, P., Dietrich C., Arand, M. et al. (2005) Aryl hydrocarbon receptor activation by cAMP vs. dioxin: Divergent signaling pathways. Proc. Natl. Acad. Sci. USA 102, 9218-9223.

45) Puga, A., Xia, Y. and Elferink, C. (2002) Role of the aryl hydrocarbon receptor in cell cycle regulation. Chem. Biol. Interact. 141, 117-130.

46) Backlund, M., Johansson, I., Mkrtchian, S. and Ingelman-Sundberg, M. (1997) Signal transductionmediated activation of the aryl hydrocarbon receptor in rat hepatome HIIE. J. Biol. Chem. 272, 31755-31763.

47) Dzeletonic, N., Mcguire, M., Daujat, J., Tholander, J., Ema, M., Fujii-Kuriyama, Y. et al. (1997) Regulation of dioxin receptor function by omeprazole. J. Biol. Chem. 272, 12705-12713.

48) Backlund, M. and Ingelman-Sundberg, M. (2005) Regulation of aryl hydrocarbon receptor signal transduction by protein tyrosine kinase. Cell Signal. 17, 39-48.

49) Whitlock Jr., J.P. (1999) Induction of cytochrome P4501A1. Annu. Rev. Pharmacol. Toxicol. 39, 103-125.

50) Yanagida, A., Sogawa, K. and Fujii-Kuriyama, Y. (1990) A novel cis-acting DNA element required for a high level of inducible expression of the rat P-450c gene. Mol. Cell. Biol. 10, 1470-1475.
51) Kobayashi, A., Sogawa, K. and Fujii-Kuriyama, Y. (1996) Cooperative interaction between $\mathrm{AhR}$ and Sp1 for drug-inducible expression of CYP1A1 gene. J. Biol. Chem. 271, 12310-12316.

52) Fujii-Kuriyama, Y. and Mimura, J. (2005) Molecular mechanisms of AhR functions in the regulation of cytochome P450 genes. Biochem. Biophys. Res. Commun. 338, 311-317.

53) Morgan, J.E. and Whitlock Jr., J.P. (1992) Transcription-dependent and transcription-independent nucleosome disruption induced by dioxin. Proc. Natl. Acad. Sci. USA 89, 11622-11626.

54) Hankinson, O. (2005) Role of coactivator in transcription activation by the aryl hydrocarbon receptor. Arch. Biochem. Biophys. 433, 379-386.

55) Beischlag, T.V., Taylor, R.T., Rose, D.W., Yoon, D., Chen, Y., Lee W.H. et al. (2004) Recruitment of thyroid hormone receptor/retinoblastoma-interacting protein 230 by the aryl hydrocarbon receptor nuclear translocator is required for the transcription response to dioxin and hypoxia. J. Biol. Chem. 279, 54620-54628.

56) Wong, S., Ge, K., Roeder, R.G. and Hankinson, O. (2004) Role of mediator in transcripotion activation by aryl hydrocarbon receptor. J. Biol. Chem. 279, 13593-13600.

57) Davarinos, N. and Pollenz, R.S. (1999) Aryl hydrocabon receptor imported into nucleus following ligand binding is rapidly degraded via the cytoplasmic proteasome following nuclear export. J. Biol. Chem. 274, 28704-28715.

58) Pollenz, R.S. and Dougherty, E.J. (2005) Redefining the role of XAP2 and CHIP in the degradation of endogenous AHR in cell culture model. J. Biol. Chem. 280, 33346-33356.

59) Lee, M.J., Peet, D.J. and Whitelaw, M. (2003) Defining the role of XAP2 in stabilization of the dioxin receptor. J. Biol. Chem. 278, 35878-35888.

60) Ohtake, F., Baba, A., Takada, I., Okada, M., Iwasaki, K., Miki, H. et al. (2007) Dioxin receptor is a ligand-dependent E3 ubiqutin ligase. Nature 446, $562-566$

61) Kawajiri, K., Kobayashi, Y., Ohtake, F., Ikuta, T., Matsushita, Y., Mimura, J. et al. (2009) Aryl hydrocarbon receptor suppresses intestinal carcinogenesis in $A P C^{\text {min/+ }}$ mice with natural ligands. Proc. Natl. Acad. Sci. USA 106, 13481-13486.

62) Yamashita, T., Ohneda, K., Nagano, M., Iemitsu, M., Makino, Y., Tanaka, H. et al. (2007) Abnormal heart development and lung remodeling in mice lacking the hypoxia-inducible factor-related basic helix-loop-helix PAS protein NEPAS. Mol. Cell. Biol. 28, 1285-1297.

63) Mimura, J., Ema, M., Sogawa, K. and FujiiKuriyama, Y. (1999) Identification of a novel mechanism of regulation of $\mathrm{Ah}$ (dioxin) receptor function. Genes Dev. 13, 20-25.

64) Baba, T., Mimura, J., Grading, K., Kuroiwa, A., Watanabe, T., Matsuda, Y. et al. (2001) Structure and expression of the Ah receptor repressor gene. J. Biol. Chem. 276, 33101-33110.

65) Oshima, M., Mimura, J., Yamamoto, M. and Fujii- 
Kuriyama, Y. (2007) Molecular mechanism of transcriptional repression of $\mathrm{AhR}$ repressor involving ANKRA2, HDAC4 and HDAC5. Bichem. Biophys. Res. Commun. 364, 276-282.

66) Oshima, M., Mimura, J., Sekine, H., Okawa, H. and Fujii-Kuriyama, Y. (2009) SUMO modification regulates the transcriptional repressor function of aryl hydrocarbon receptor repressor. J. Biol. Chem. 284, 11017-11026.

67) Zudaire, E., Cuesta, N., Murty, V., Woodson, K., Adams, L., Gonzalez, N. et al. (2008) The aryl hydrocarbon receptor repressor is a putative tumor suppressor in multiple human cancers. J. Clin. Invest. 118, 640-650.

68) Kim, S.H., Choi, Y.M., Lee, G.H., Hong, M.A., Lee, K.S., Lee, B.S. et al. (2007) Association between susceptibility to advanced stage endometoriosis and the genetic polymorphisms of aryl hydrocarbon receptor repressor and glutathione-S-transferase T1 genes. Hum. Reprod. 22, 1866-1870.

69) Watanabe, T., Imoto, I., Kosugi, Y., Fukuda, Y., Mimura, J., Fujii, Y. et al. (2001) Human aryl hydrocarbon receptor repressor: Genomic structure and analysis of polymorphisms in endometoriosis. J. Hum. Genet. 46, 342-346.

70) Watanabe, M., Sueoka, K., Sasagawa, I., Nakabayashi, A., Yoshimura, Y. and Ogata, T. (2004) Association of male infertility with Pro185Ala polymorphism in aryl hydrocabon receptor repressor gene: Implication for susceptibility to dioxins. Fertil. Steril. 82, 1067-1071.

71) Safe, S., Wormke, M. and Sumudio, I. (2000) Mechanisms of inhibitory aryl hydrocarbon receptorestrogen receptor crosstalk in human breast cancer cells. J. Mammary Gland Biol. Neoplasia 5, 295306.

72) Ohtake, F., Takeyama, K., Matsumoto, T., Kitagawa, H., Yamamoto, Y., Nohara, K. et al. (2003) Modulation of oestrogen receptor signaling by association with the activated dioxin receptor. Nature 423, 545-550.

73) Kharat, I. and Saatiagli, F. (1996) Antiestrogenic effects of 2,3,7,8-tetrachlorodibenzo- $p$-dioxin are mediated by direct transcriptional interference with liganded estrogen receptor. Cross-talk between aryl hydrocarbon- and estrogen-mediated signaling. J. Biol. Chem. 271, 10533-10537.

74) Valley, C.C., Metivier, R., Solodin, M.M., Fowler, A.M., Mashek, M.T., Hill, L. and Alarid, E.T. (2005) Differentioal regulation of oestrogen-inducible proteolysis and transcription by the oestrogen receptor alpha N-terminus. Mol. Cell. Biol. 25, 5417-5428.

75) McKenna, N.J. and O'Malley, B.W. (2002) Combinatorial control of gene expression by nuclear receptor and coregulator. Cell 108, 464-474.

76) Reid, G., Denger, S., Kos, M. and Gannon, F. (2002) Human estrogen receptor-alpha: Regulation, modification and degradation. Cell. Med. Life Sci. 59, 821-831.

77) Su, L.K., Vogelstein, B. and Kinzler, K.W. (1993) Association of the APC tumor suppressor protein with catenins. Science 262, 1734-1737.
78) Yang, J., Zhang, W., Evans, P.M., Chen, X., He, X. and Liu, C. (2006) Adenomatous polyposis coli (APC) differentially regulates $\beta$-catenin phosphorylation and ubiquitylation in colon cancer. J. Biol. Chem. 281, 17751-17757.

79) Wattenberg, L. (1985) Chemoprevention of cancer. Cancer Res. 45, 1-8.

80) Xu, M., Bailey, A.C., Hernaez, J.F., Taoka, C.R., Shut, H.A. and Dashwood, R.H. (1996) Protection by green tea, black tea, and indole-3-carbinol against 2-amino-3-methylimidazo[4,5- $f$ ]quinoline-induced DNA adducts and colonic aberrant crypts in the F344 rat. Carcinogenesis 17, 1429-1434.

81) Chen, I., McDougal, A., Wang, F. and Safe, S. (1998) Aryl hydrocarbon receptor-mediated antiestrogenic and anti-tumorigenic activity of diindolylmethane. Carcinogenesis 19, 1631-1639.

82) Hernandez-Ochoa, I., Karman, B.N. and Flaws, J.A. (2009) The role of the aryl hydrocarbon receptor in the female reproduction system. Biochem. Pharmacol. 77, 547-559.

83) Abbott, B.D., Schmid, J.E., Pitt, J.A., Buckalew, A.R., Wood, C.R., Held, G.A. and Diliberto, J.J. (1999) Adverse reproductive outcomes in transgenic Ah receptor deficient mouse. Toxicol. Appl. Pharmacol. 155, 62-70.

84) Baba, T., Mimura, J., Nakamura, N., Harada, N., Yamamoto, M., Morohashi, K. and Fujii-Kuriyama, Y. (2005) Intrinsic function of the aryl hydrocarbon (dioxin) receptor as a key factor in female reproduction. Mol. Cell. Biol. 25, 10040-10051.

85) Channing, C.P., Schaerf, F.W., Anderson, L.D. and Tsafriri, A. (1980) Ovarian follicular and luteal physiology. Int. Rev. Physiol. 22, 117-201.

86) Bennett, J.C., Lin, T.M., Loeffler, I.K., Peterson, R.E. and Flaws, J.A. (2000) Physiological role of the aryl hydrocarbon receptor in mouse ovary development. Toxicol. Sci. 56, 382-388.

87) Barnett, K.R., Tomic, D., Gupta, R.K., Babus, J.K., Roby, K.F., Terranova, P.F. and Flaws, J.A. (2007) The aryl hydrocarbon receptor is required for normal gonadotropin responsiveness in the mouse ovary. Toxicol. Appl. Pharmacol. 223 66-77.

88) Drummond, A.E. (2006) The role of steroids in follicular growth. Reprod. Biol. Endocrinol. 4, 16-26.

89) Foxcroft, G.R. and Hunter, M.G. (1985) Basic physiology of follicular maturation in the pig. J. Reprod. Fertil. Suppl. 33, 1-19.

90) Danadeau, F.X. and Ascoli, M. (2005) The differential effects of the gonadotropin receptors on aromatase expression in primary cultures of immature rat granulose cells are highly dependent on the density of receptors expressed and the activation of the inositol phosphate cascade. Endocrinology 146, 3907-3916.

91) Barnett, K.R., Tomic, D., Gupta, R.K., Miller, K.P., Meachum, S., Paulose, T. and Flaws, J.A. (2007) The aryl hydrocarbon receptor affects mouse ovarian follicle growth via mechanisms involving estradiol regulation and responsiveness. Biol. Reprod. 76, 1062-1070.

92) Fisher, C.R., Graves, K.H., Parlow, A.F. and Simp- 
son, E.R. (1998) Characterization of mice deficient in aromatase (ArKO) because of targeted disruption of cyp1a1 gene. Proc. Natl. Acad. Sci. USA 95, 6965-6970.

93) Dupont, S., Krust, A., Gransmuller, A., Dierich, P., Chambon, P. and Mark, M. (2000) Effect of single and compound knockout of estrogen receptors alpha (ER alpha) and beta (ER beta) on mouse reproduction phenotypes. Development 127, 42774291.

94) Kitajima, M., Khan, K.N., Fujita, A., Masuzaki H., Koji, T. and Ishimaru, T. (2004) Expression of the aryl hydrocarbon receptor in the peri-implantation period of the mouse uterus and the impact of dioxin on mouse implantation. Arch. Histol. Cytol. 67, 465-474.

95) Hushka, L.J., Williams, J.S. and Greenlee, W.F. (1998) Characterization of 2,3,7,8-tetrachlorodibenzofuran-dependent suppression and AH receptor pathway gene expression in the developing mouse mammary gland. Toxicol. Appl. Pharmacol. 152, 200-210.

96) Kerkvliet, N.I. (1995) Immunological effects of chlorinated dibenzo- $p$-dioxins. Environ. Health Perspect. 103 (Suppl. 9), 47-53.

97) Kerkvliet, N.I. (2009) AHR-mediated immunomodulation: The role of altered gene transcription. Biochem. Pharmacol. 77, 746-760.

98) Stevens, E.A., Mezrich, J.D. and Bradfield, C.A. (2009) The aryl hydrocarbon receptor A perspective on potential roles in the immunesystem. Immunol. 127, 299-311.

99) Clark, G.C., Taylor, M.J., Tritscher, A.M. and Lucier, G.W. (1991) Tumor necrosis factor involvement in 2,3,7,8-tetrachlorodibenzo- $p$-dioxin-mediated endotoxin hypersensitivity in C57BL/6J mice conjenic at Ah locus. Toxicol. Appl. Pharmacol. 111, 422-431.

100) Moos, A.B., Oughton, J.A., Kerkvliet, N.I. (1997) the effects of 2,3,7,8-tetrachlorodibenzo- $p$-dioxin (TCDD) on tumor necrosis factor (TNF) production by peritoneal cells. Toxicol. Lett. 90, 145-153.

101) Kimura, A., Naka, T., Nakamura, T., Chinen, I., Masuda, K., Nohara, K. et al. (2009) Aryl hydrocarbon receptor in combination with Stat1 regulates LPS-induced inflammatory responses. J. Exp. Med. 206, 2027-2035.
102) Sekine, H., Mimura, J., Oshima, M., Okawa, H., Kanno, J., Igarashi, K. et al. (2009) Hypersensitivity of AhR-deficient mice to LPS-induced septic shock. Mol. Cell. Biol. (in press).

103) Greten, F.R., Arkan, M.C., Bollrath, J., Hsu, L.C.,

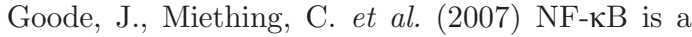
negative regulator of IL-1 $\beta$ as revealed by genetic and pharmacological inhibitor of IKK $\beta$. Cell 130, 918-931.

104) Vogel, C.F., Sciullo, E., Li, W., Wong, P., Lazennec, G. and Matsumura, F. (2007) RelB, a new partner of aryl hydrocarbon receptor-mediated transcription. Mol. Endocrinol. 21, 2941-2955.

105) Funatake, C.J., Marshall, N.B., Steppan, L.B., Mourich, D.V. and Kerkvliet, N.I. (2005) Cutting edge: activation of the aryl hydrocarbon receptor by $2,3,7,8$-tetrachlorodibenzo- $p$-dioxin generates a population of $\mathrm{CD} 4^{+} \mathrm{CD} 25^{+}$cells with characteristics of regulatory $\mathrm{T}$ cells. J. Immunol. 175, 41844188.

106) Hauben, E., Gregori, S., Draghici, E., Magliavacca, B., Olivieri, S., Voisetschlager, M. and Poncarolo, M.G. (2008) Activation of aryl hydrocarbon receptor promotes allograft-specific tolerance through direct and dendritic cell-mediated effects on regulatory T cell. Blood 112, 1214-1222.

107) Negishi, T., Kato, Y., Ooneda, O., Mimura, J., Takada, T., Mochzuki, H. et al. (2005) Effect of aryl hydrocarbon receptor signal on modulation of Th1/Th2 balance. J. Immunol. 175, 7348-7356.

108) Quintana, F.J., Basso, A.S., Inglesias, A.H., Korn, T., Farez, M.F., Bettelli, E. et al. (2008) Control of Treg and Th17 cell differentiation by the aryl hydrocarbon receptor. Nature 453, 65-71.

109) Veldhoen, M., Hirata, K., Westendorf, A.M., Bauer, J., Dumoutier, L., Renauld, J.C. and Stockinger, B. (2008) The aryl hydrocarbon receptor Links Th17cell-mediated autoimmunity to environmental toxins. Nature 453, 106-109.

110) Kimura, A., Naka, T., Nohara, K., Fujii-Kuriyama, F. and Kishimoto, T. (2008) Aryl hydrocarbon receptor regulates Stat1 activation and participates in the development of Th17 cells. Proc. Natl. Acad. Sci. USA 105, 9721-9726.

(Received Sept. 24, 2009; accepted Nov. 9, 2009) 


\section{Profile}

Yoshiaki Fujii (-Kuriyama) was born in 1938 and after graduating from Faculty of Science and the Master Course of the University of Tokyo, he jointed Sankyo Pharmaceutical Co., Central Research Laboratories in 1965. He received the degree of Doctor of Science from Osaka University in 1970 and moved to Kansai Medical University as lecturer in 1975. Then, he jointed Cancer Institute, Japan Foundation for Cancer Research as associate member in 1977 and subsequently as member. From studies on the xenobiotic metabolism and metabolic activation of chemical carcinogens, he was the first to elucidate the primary structure of drug-metabolizing P450 and paved the way to molecular biological studies on P450 which shows a extremely broad distribution in living organisms from bacteria to plants and animals by clarifying the primary

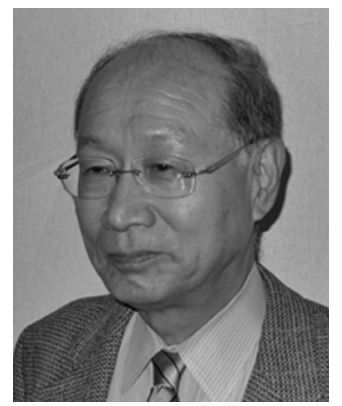
structures of other drug-metabolizing P450s and steroidogenic P450s, and their structure/function relationship. The P450 super-family is now known to consist of more than 6000 molecular species in nature which are involved in vast varieties of functions ranging from drug metabolism, steroidogenesis, synthesis of many lipophilic bioactive compounds including prostaglandins and gibberellin to flower coloring. He was promoted to Professor of Tohoku University in 1987 and continued to work until his retirement as Professor Emeritus in 2002. From his work on the induction mechanisms of drug-metabolizing P450, he identified AhR as a transcription factor working in response to $\mathrm{PAH}$ and was the first to solve the primary structure of AhR, leading to elucidation of the induction mechanisms of P450 and its involvement in teratogenesis and carcinogenesis caused by dioxins. With his recent finding that AhR acts as an E3 ubiqutin ligase, he demonstrated that AhR functions as a multiregulatory factor in drug metabolism, innate immunity and colonic tumor suppression. After serving as Guest Professor in University of Tsukuba from 2002 to 2009, he is Guest Professor in Medical Research Institute, Tokyo Medical and Dental University and Visiting Scientist in Institute of Molecular Cellular Biosciences, The University of Tokyo. He was awarded the Princess Takamatsu Cancer Prize in 2006. 